\title{
Polyphasic characterization of rhizobia that nodulate Phaseolus vulgaris in West Africa (Senegal and Gambia)
}

\author{
Adama Diouf, ${ }^{1}$ Philippe de Lajudie, ${ }^{2}$ Marc Neyra, ${ }^{1}$ Karel Kersters, ${ }^{3}$ \\ Monique Gillis, ${ }^{3}$ Esperanza Martinez-Romero $^{4}$ and Mamadou Gueye ${ }^{1}$
}

Author for correspondence: Mamadou Gueye. Tel: +221 84933 21. Fax: +221 8321675. e-mail: mamadou.gueye@orstom.sn

1 MIRCEN/Centre ISRA-IRD, BP 1386, Dakar, Senegal, West Africa

2 Laboratoire des Symbioses Tropicales et Méditerranéennes, IRD, Campus de Baillarguet, BP 5035, 34032 Montpellier Cédex 1, France

3 Laboratorium voor Microbiologie, Universiteit Gent, K.-L. Ledeganckstraat 35 B-9000 Ghent, Belgium

4 Centro de Investigación sobre Fijación de Nitrógeno, Universidad Nacional Autónoma de México, AP 565-A Cuernavaca, Morelos, Mexico

\begin{abstract}
Fifty-eight new isolates were obtained from root nodules of common bean (Phaseolus vulgaris) cultivated in soils originating from different agroecological areas in Senegal and Gambia (West Africa). A polyphasic approach including both phenotypic and genotypic techniques was used to study the diversity of the $\mathbf{5 8}$ Rhizobium isolates and to determine their taxonomic relationships with reference strains. All the techniques performed, analysis of multilocus enzyme electrophoretic patterns, SDS-PAGE profiles of total cell proteins, PCR-RFLP analysis of the genes encoding 16S rRNA and of the 16S-23S RNA intergenic spacer region (ITS-PCR-RFLP), auxanographic tests using API galleries and nodulation tests lead to the consensus conclusion that the new rhizobial isolates formed two main distinct groups, I and II, belonging to Rhizobium tropici type B and Rhizobium etli, respectively. By MLEE R. etli and group II strains showed several related electrophoretic types, evidencing some extent of internal heterogeneity among them. This heterogeneity was confirmed by other techniques (ITS-PCR-RFLP, SDS-PAGE and host-plantspecificity) with the same nine distinct strains of group II showing some differences from the core of group II (54 strains).
\end{abstract}

Keywords: Rhizobium, root nodules, common bean (Phaseolus vulgaris)

\section{INTRODUCTION}

During recent years the classification of rhizobia that nodulate common bean (Phaseolus vulgaris) plants has been progressively revised as more rhizobial diversity is gradually discovered in different parts of the world (Martínez-Romero et al., 1991; Segovia et al., 1993; Amarger et al., 1997). These rhizobia were initially assigned to Rhizobium leguminosarum bv. phaseoli on the basis of their host specificity, separate from $R$. leguminosarum bv. viciae and $R$. leguminosarum bv. trifolii, symbionts of peas (Pisum spp., Vicia spp.) and clovers (Trifolium spp.) respectively (Jordan, 1984). This subdivision was also supported by their different symbiotic plasmids, encoding the nodulation specificities (Martínez et al., 1985, 1988). R. leguminosarum bv. phaseoli was long recognized to be taxonomically heterogeneous as evidenced by protein profile analysis

Abbreviations: ET, electrophoretic type; ITS, internal transcribed spacer; MLEE, multilocus enzyme electrophoresis.
(Roberts et al., 1980), multilocus enzyme electrophoresis (MLEE) (Piñero et al., 1988; Eardly et al., 1995), DNA relatedness analysis (Laguerre et al., 1993; van Berkum et al., 1996) and 16S rRNA genes (rDNA) sequencing (Segovia et al., 1993; Laguerre et al., 1993; Hernandez-Lucas et al., 1995). R. leguminosarum bv. phaseoli from Mexico and South America was first divided into $R$. leguminosarum bv. phaseoli type I and type II (Martínez et al., 1988). Rhizobium tropici types $\mathrm{A}$ and $\mathrm{B}$ were first proposed for type II strains carrying a single nifH gene copy. $R$. tropici types $\mathrm{A}$ and $\mathrm{B}$ are distinguished by their DNA-DNA hybridization values, a number of phenotypic characteristics and the presence of a specific megaplasmid (Martínez-Romero et al., 1991; Geniaux et al., 1995). Rhizobium etli was then proposed for R. leguminosarum bv. phaseoli type I strains which contain multiple copies of the nitrogenase reductase gene (nifH) on their symbiotic plasmids (Martínez et al., 1985; Segovia et al., 1993). More recently, two additional groups of Rhizobium strains nodulating bean plants were characterized in European soils and proposed as two new species, 
Rhizobium gallicum and Rhizobium giardinii (Amarger et al., 1997). Phaseolus vulgaris is commonly reported to originate from America whereas its symbiotic rhizobial partners are thought to be diverse and widely spread around the world. Native rhizobia nodulating common bean in African soils have been reported to be taxonomically related to $R$. tropici in East and South Africa (Anyango et al., 1995; Dagutat \& Steyn, 1995) and to $R$. tropici and $R$. etli in Central Africa (Tjahjoleksono, 1993).

In this paper, we studied the diversity and taxonomic relationships among 58 rhizobial strains originating from different agroecological areas in Senegal and Gambia. Using techniques with various discriminative powers such as physiological and auxanographic tests, host specificity, MLEE, analysis of total cell protein profiles by SDS-PAGE, RFLP analysis of PCRamplified ITS (internal transcribed spacer) $16 \mathrm{~S}-23 \mathrm{~S}$ and $16 \mathrm{~S}$ rDNA we showed that, in West Africa, Phaseolus vulgaris is naturally nodulated by Rhizobium strains belonging to $R$. tropici type $\mathrm{B}$ and $R$. etli.

\section{METHODS}

Bacterial strains. Rhizobial strains (Table 1) were isolated from root nodules of common bean cultivated during 15-20 d either in field conditions or in pots containing soils sampled at 5-20 cm depth in different places in Senegal and Gambia.

These rhizobial strains were compared to several reference strains representing the different Rhizobium, Sinorhizobium, Mesorhizobium and Agrobacterium species or groups.

Growth and culture conditions. New isolates and reference Rhizobium, Sinorhizobium and Mesorhizobium strains were maintained on yeast mannitol agar (YMA), containing $\left(\mathrm{g} \mathrm{l}^{-1}\right)$ : mannitol, 10; sodium glutamate, $0 \cdot 5 ; \mathrm{K}_{2} \mathrm{HPO}_{4}, 0 \cdot 5$; $\mathrm{MgSO}_{4} .7 \mathrm{H}_{2} \mathrm{O}, 0 \cdot 2 ; \mathrm{NaCl}, 0.05 ; \mathrm{CaCl}_{2}, 0.04 ; \mathrm{FeCl}_{3}, 0.004$; yeast extract, 1; pH 6.8; agar, 20. Agrobacterium strains were maintained on yeast extract peptone-glucose medium,

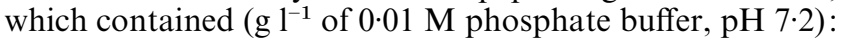
peptone, 5 ; yeast extract, 1 ; beef extract, 5 ; sucrose, 5 and $\mathrm{MgSO}_{4} \cdot 7 \mathrm{H}_{2} \mathrm{O}, 0.592$.

Morphological and physiological tests. Cell dimensions and morphology were determined by phase-contrast microscopy. Growth of strains was also studied on Luria broth (LB) medium containing $\left(\mathrm{g}^{-1}\right)$ : bacto-tryptone, 50 ; yeast extract, 5 ; sodium chloride, 5 and on peptone yeast extract (PY) medium containing $\left(\mathrm{g}^{-1}\right)$ : peptone, 5 ; yeast extract, 3 ; supplemented with $10 \mathrm{ml}$ of sterile solution of $\mathrm{CaCl}_{2} 0.7 \mathrm{M}$. The maximum growth temperature on YM medium was determined for all strains.

PCR/RFLP of 16S-23S rDNA ITS region. Bacterial genomic DNA was extracted and purified as described by Boucher et al. (1987). Primers FGPS 1490, corresponding to positions 1521-1541 of Escherichia coli (Navarro et al., 1992), and FGPL 132', corresponding to positions 114-132 of E. coli (Ponsonnet \& Nesme, 1994), were used for PCR amplification. PCR was carried out in a $100 \mu$ reaction volume by mixing $2 \mu \mathrm{l}$ DNA extract with the polymerase reaction buffer $(10 \mathrm{mM}$ Tris $/ \mathrm{HCl}, \mathrm{pH} 8 \cdot 3 ; 50 \mathrm{mM} \mathrm{KCl}, 0.0 .1 \%$ gelatin, $2 \mathrm{mM} \mathrm{MgCl}_{2}$ ); Taq polymerase (Bioprobe), $0 \cdot 8 \mu \mathrm{l}$ (5 $\mathrm{U} /$ reaction); $2 \mu \mathrm{l}$ each of the $10 \mu \mathrm{M}$ dNTPs (dATP, dCTP,
dGTP, dTTP), $1 \mu$ l each primer $(0 \cdot 05 \mu \mathrm{M})$. PCR amplification was performed in a Gene Amp PCR System 2400 thermal cycler adjusted to the following temperature profile: initial denaturation at $94^{\circ} \mathrm{C}$ for $5 \mathrm{~min}$; 35 amplification cycles (denaturation at $94{ }^{\circ} \mathrm{C}$ for $1 \mathrm{~min}$, hybridization of primers at $55^{\circ} \mathrm{C}$ for $1 \mathrm{~min}$ and extension at $72{ }^{\circ} \mathrm{C}$ for $1.5 \mathrm{~min}$ ); final extension at $72{ }^{\circ} \mathrm{C}$ for $5 \mathrm{~min}$. Amplification was checked by horizontal agarose $(1 \%$, w/v) gel electrophoresis using $10 \mu \mathrm{l}$ of the PCR product.

Aliquots $(8 \mu \mathrm{l})$ of PCR products were digested with the following restriction endonucleases (5 U/reaction): AluI, DdeI, HhaI, PalI (Pharmacia), HinfI, MspI (Gibco-BRL), RsaI and TaqI (Boehringer Mannheim). Restricted DNA was analysed by horizontal agarose (NuSieve 3:1, FMC $3 \%, \mathrm{w} / \mathrm{v}$ ) gel electrophoresis. Electrophoresis was carried out at $60 \mathrm{~V}$ for $4 \mathrm{~h}$ and gels were stained as described above and photographed under UV illumination with Polaroid 665 positive/negative film.

MLEE. Isolates and reference strains were grown overnight on an orbital shaker incubator at $30^{\circ} \mathrm{C}$ in $30 \mathrm{ml} \mathrm{PY}$ medium. Cells were harvested by centrifugation at $6000 \mathrm{~g}$ for $10 \mathrm{~min}$ at $4{ }^{\circ} \mathrm{C}$. After suspension in $0.2 \mathrm{ml} \mathrm{MgSO}_{4} 10 \mathrm{mM}$, the bacteria were lysed by adding $40 \mu \mathrm{l}$ of a lysozyme solution (10 mg ml ${ }^{-1} \mathrm{MgSO}_{4} 10 \mathrm{mM}$ ). The lysate maintained on ice was then placed for $15 \mathrm{~min}$ at room temperature and stored at $-70{ }^{\circ} \mathrm{C}$ for $10 \mathrm{~min}$ before use.

Techniques of starch gel electrophoresis and selective staining of enzymes were performed as described by Selander et al. (1986). The electrophoretic buffer system, Tris-citrate ( $\mathrm{pH}$ 8.0) was used for the following enzymes assayed: malate dehydrogenase, isocitrate dehydrogenase, glucose-6-phosphate dehydrogenase, glutamate dehydrogenase, xanthine dehydrogenase, phosphoglucomutase, esterases, aconitase, indophenol oxidase (super oxide dismutase), hexokinase and alanine dehydrogenase.

Distinctive mobility variants (electromorphs) of each enzyme, numbered in order of decreasing anodal mobility, were equated with alleles at the corresponding gene locus, and electromorph profiles for the 12 enzymes (electrophoretic types, ETs) were considered to be multilocus genotypes (Table 2).

The genetic diversity $(h)$ for an enzyme locus was calculated from allele frequencies for ETs as $h=1-\Sigma x_{\mathrm{i}}{ }^{2}(n /(n-1))$ where $x_{\mathrm{i}}$ is the frequency of the $i$ th allele and $n$ is the number of ETs. The mean genetic diversity per locus $(H)$ is the arithmetic mean of $h$ values for the 12 loci (Selander et al., 1986). The genetic distance between each pair of ETs was estimated; clustering at a matrix of pairwise genetic distances was performed by the unweighted pair group method with averages of Nei \& Li (1979).

PAGE of total bacterial proteins (SDSPAGE). All strains were grown at $28^{\circ} \mathrm{C}$ for $48 \mathrm{~h}$ in Roux flasks on tryptone yeast extract (TY) medium containing $\left(\mathrm{g} \mathrm{l}^{-1}\right)$ : tryptone extract, 5; yeast extract, $0.75 ; \mathrm{KH}_{2} \mathrm{PO}_{4}, 0.454 ; \mathrm{Na}_{2} \mathrm{HPO}_{4} .12 \mathrm{H}_{2} \mathrm{O}$, $2 \cdot 388 ; \mathrm{CaCl}_{2} \cdot 6 \mathrm{H}_{2} \mathrm{O}, 1$; agar (Lab M), 20; pH 6.8-7. Wholecell protein extracts were prepared from $80 \mathrm{mg}$ cells and SDS-PAGE was performed using slight modifications of the Laemmli (1970) procedure, as described previously (Kiredjian et al., 1986). The normalized densitometric traces of the protein electrophoretic patterns were grouped by numerical analysis, using the GelCompar software package (Vauterin \& Vauterin, 1992). The similarity between all pairs of traces was expressed by the Pearson product-moment correlation coefficient $(r)$ converted for convenience to a percentage value (Pot et al., 1989, 1994). 
Table 1. Rhizobium strains ISRA isolated from Phaseolus vulgaris and reference strains used in this study

Abbreviations: CIAT, Rhizobium Collection, Centro International de Agricultura Tropical, Cali, Columbia; CIFN, Centro de Investigación sobre Fijación de Nitrógeno, Universidad Nacional Autónoma de México, AP 565-A Cuernavaca, Morelos, Mexico; ISRA, Institut Sénégalais de la Recherche Agricole, Centre ISRA-ORSTOM, BP 1386, Dakar, Senegal;

BCCM/LMG ${ }^{\mathrm{TM}}$, Bacteria Collection, Laboratorium voor Microbiologie, K.-L. Ledeganckstraat 35, B9000 Gent, Belgium; ORSTOM, Institut Français de Recherche Scientifique pour le Développement en Coopération, BP 1386, Dakar, Senegal; USDA, US Department of Agriculture, Beltsville, MD, USA; Sn, Senegal.

\begin{tabular}{|c|c|c|c|}
\hline Strain & Host plant or origin & Geographical origin & Source or reference \\
\hline \multicolumn{4}{|l|}{ New isolates* } \\
\hline \multicolumn{4}{|l|}{ Group I } \\
\hline ISRA 350 & Phaseolus vulgaris & Bassin arachidier, $\mathrm{Sn}$ & This work \\
\hline ISRA 352 & Phaseolus vulgaris & Sylvopastorale, $\mathrm{Sn}$ & This work \\
\hline ISRA 354 & Phaseolus vulgaris & Sylvopastorale, Sn & This work \\
\hline ISRA 554 & Phaseolus vulgaris & Sylvopastorale, Sn & This work \\
\hline \multicolumn{4}{|l|}{ Group II } \\
\hline \multicolumn{4}{|l|}{ Subgroup II.1 } \\
\hline ISRA 30 & Phaseolus vulgaris & Niayes, Sn & This work \\
\hline ISRA 319 & Phaseolus vulgaris & Niayes, Sn & This work \\
\hline ISRA 351 & Phaseolus vulgaris & Bassin arachidier, $\mathrm{Sn}$ & This work \\
\hline ISRA 353 & Phaseolus vulgaris & Sylvopastorale, $\mathrm{Sn}$ & This work \\
\hline ISRA 355 & Phaseolus vulgaris & Bassin arachidier, $\mathrm{Sn}$ & This work \\
\hline ISRA 356 & Phaseolus vulgaris & Bassin arachidier, $\mathrm{Sn}$ & This work \\
\hline ISRA 357 & Phaseolus vulgaris & Sylvopastorale, Sn & This work \\
\hline ISRA 361 & Phaseolus vulgaris & Bassin arachidier, $\mathrm{Sn}$ & This work \\
\hline ISRA 362 & Phaseolus vulgaris & Sylvopastorale, $\mathrm{Sn}$ & This work \\
\hline ISRA 363 & Phaseolus vulgaris & Bassin arachidier, $\mathrm{Sn}$ & This work \\
\hline ISRA 364 & Phaseolus vulgaris & Bassin arachidier, $\mathrm{Sn}$ & This work \\
\hline ISRA 365 & Phaseolus vulgaris & Bassin arachidier, $\mathrm{Sn}$ & This work \\
\hline ISRA 553 & Phaseolus vulgaris & Bassin arachidier, $\mathrm{Sn}$ & This work \\
\hline ISRA 555 & Phaseolus vulgaris & Bassin arachidier, $\mathrm{Sn}$ & This work \\
\hline ISRA 556 & Phaseolus vulgaris & Sylvopastorale, $\mathrm{Sn}$ & This work \\
\hline ISRA 557 & Phaseolus vulgaris & Sylvopastorale, Sn & This work \\
\hline ISRA 558 & Phaseolus vulgaris & Sylvopastorale, Sn & This work \\
\hline ISRA 559 & Phaseolus vulgaris & Fleuve, $\mathrm{Sn}$ & This work \\
\hline ISRA 561 & Phaseolus vulgaris & Fleuve, Sn & This work \\
\hline ISRA 562 & Phaseolus vulgaris & Fleuve, Sn & This work \\
\hline ISRA 563 & Phaseolus vulgaris & Fleuve, Sn & This work \\
\hline ISRA 564 & Phaseolus vulgaris & Bassin arachidier, $\mathrm{Sn}$ & This work \\
\hline ISRA 566 & Phaseolus vulgaris & Fleuve, $\mathrm{Sn}$ & This work \\
\hline ISRA 567 & Phaseolus vulgaris & Bassin arachidier, $\mathrm{Sn}$ & This work \\
\hline ISRA 568 & Phaseolus vulgaris & Fleuve, $\mathrm{Sn}$ & This work \\
\hline ISRA 569 & Phaseolus vulgaris & Fleuve, Sn & This work \\
\hline ISRA 570 & Phaseolus vulgaris & Fleuve, Sn & This work \\
\hline ISRA 571 & Phaseolus vulgaris & Fleuve, Sn & This work \\
\hline ISRA 572 & Phaseolus vulgaris & Bassin arachidier, $\mathrm{Sn}$ & This work \\
\hline ISRA 573 & Phaseolus vulgaris & Bassin arachidier, $\mathrm{Sn}$ & This work \\
\hline ISRA 574 & Phaseolus vulgaris & Fleuve, $\mathrm{Sn}$ & This work \\
\hline ISRA 575 & Phaseolus vulgaris & Fleuve, $\mathrm{Sn}$ & This work \\
\hline ISRA 576 & Phaseolus vulgaris & Fleuve, $\mathrm{Sn}$ & This work \\
\hline ISRA 577 & Phaseolus vulgaris & Fleuve, Sn & This work \\
\hline ISRA 578 & Phaseolus vulgaris & Fleuve, Sn & This work \\
\hline ISRA 579 & Phaseolus vulgaris & Fleuve, $\mathrm{Sn}$ & This work \\
\hline ISRA 580 & Phaseolus vulgaris & Gambia & This work \\
\hline
\end{tabular}


Table 1 (cont.)

\begin{tabular}{|c|c|c|c|}
\hline Strain & Host plant or origin & Geographical origin & Source or reference \\
\hline ISRA 581 & Phaseolus vulgaris & Gambia & This work \\
\hline ISRA 582 & Phaseolus vulgaris & Gambia & This work \\
\hline ISRA 583 & Phaseolus vulgaris & Gambia & This work \\
\hline ISRA 584 & Phaseolus vulgaris & Gambia & This work \\
\hline ISRA 585 & Phaseolus vulgaris & Gambia & This work \\
\hline ISRA 586 & Phaseolus vulgaris & Bassin arachidier, $\mathrm{Sn}$ & This work \\
\hline ISRA 587 & Phaseolus vulgaris & Bassin arachidier, $\mathrm{Sn}$ & This work \\
\hline ISRA 588 & Phaseolus vulgaris & Bassin arachidier, $\mathrm{Sn}$ & This work \\
\hline \multicolumn{4}{|l|}{ Subgroup II.2 } \\
\hline ISRA 21 & Phaseolus vulgaris & Casamance, $\mathrm{Sn}$ & This work \\
\hline ISRA 77 & Phaseolus vulgaris & Casamance, $\mathrm{Sn}$ & This work \\
\hline ISRA 78 & Phaseolus vulgaris & Casamance, $\mathrm{Sn}$ & This work \\
\hline ISRA 560 & Phaseolus vulgaris & Fleuve, $\mathrm{Sn}$ & This work \\
\hline ISRA 565 & Phaseolus vulgaris & Fleuve, Sn & This work \\
\hline \multicolumn{4}{|l|}{ Subgroup II.3 } \\
\hline ISRA 27 & Phaseolus vulgaris & Niayes, Sn & This work \\
\hline ISRA 59 & Phaseolus vulgaris & Niayes, Sn & This work \\
\hline ISRA 61 & Phaseolus vulgaris & Niayes, $\mathrm{Sn}$ & This work \\
\hline ISRA 69 & Phaseolus vulgaris & Niayes, Sn & This work \\
\hline \multicolumn{4}{|c|}{ Reference strains } \\
\hline \multicolumn{4}{|c|}{ Rhizobium tropici } \\
\hline \multicolumn{4}{|l|}{ Type A } \\
\hline $\mathrm{CFN} 299^{\mathrm{T}}$ & Phaseolus vulgaris & Brazil & $\begin{array}{l}\text { Martinez-Romero et al. } \\
\text { (1991) }\end{array}$ \\
\hline LMG 9502 & Phaseolus vulgaris & & LMG \\
\hline \multicolumn{4}{|l|}{ Type B } \\
\hline CIAT $899^{\mathrm{T}}$ & Phaseolus vulgaris & Colombia & $\begin{array}{l}\text { Martinez-Romero et al. } \\
\text { (1991) }\end{array}$ \\
\hline LMG 9519 & Phaseolus vulgaris & & LMG \\
\hline LMG 9518 & Phaseolus vulgaris & & LMG \\
\hline \multicolumn{4}{|l|}{ Rhizobium etli } \\
\hline $\mathrm{CFN} 42^{\mathrm{T}}$ & Phaseolus vulgaris & Mexico & Segovia et al. (1993) \\
\hline BRA 5 & Phaseolus vulgaris & Brazil & CIFN \\
\hline F6 & Phaseolus vulgaris & Mexico & CIFN \\
\hline F8 & Phaseolus vulgaris & Mexico & CIFN \\
\hline F16 & Phaseolus vulgaris & Mexico & CIFN \\
\hline Viking 1 & Phaseolus vulgaris & & Segovia et al. (1993) \\
\hline USDA 9041 & Phaseolus vulgaris & & \\
\hline USDA 2667 & Phaseolus vulgaris & & \\
\hline \multicolumn{4}{|c|}{$\begin{array}{l}\text { Rhizobium leguminosarum } \\
\text { biovar phaseoli }\end{array}$} \\
\hline LMG 8820 & Phaseolus vulgaris & & LMG \\
\hline biovar viciae & & & \\
\hline USDA 2370 & Pisum sativum & & CIFN \\
\hline biovar trifoli & & & \\
\hline LMG 8819 & Trifolium pratense & & LMG \\
\hline \multicolumn{4}{|c|}{ Rhizobium leguminosarum } \\
\hline LMG 9505 & & & LMG \\
\hline LMG 6122 & Trifolium repens & Australia & LMG \\
\hline LMG 9504 & & & LMG \\
\hline $316 \mathrm{C} 10 \mathrm{~A}$ & & & \\
\hline
\end{tabular}


Table 1 (cont.)

\begin{tabular}{|c|c|c|c|}
\hline Strain & Host plant or origin & Geographical origin & Source or reference \\
\hline \multicolumn{4}{|c|}{ Rhizobium galegae } \\
\hline LMG $6214^{\mathrm{T}}$ & Galega orientalis & Finland & LMG \\
\hline LMG 6215 & Galega orientalis & USSR & LMG \\
\hline \multicolumn{4}{|l|}{ Rhizobium sp. } \\
\hline ORS 240 & Pterocarpus lucens & Senegal & ORSTOM \\
\hline ORS 1181 & Acacia senegal & Senegal & ORSTOM \\
\hline ORS 248 & Pterocarpus lucens & Senegal & ORSTOM \\
\hline \multicolumn{4}{|c|}{ Mesorhizobium plurifarium } \\
\hline ORS 1001 & Acacia senegal & Senegal & de Lajudie et al. (1998) \\
\hline ORS 1004 & Acacia senegal & Senegal & de Lajudie et al. (1998) \\
\hline ORS 1002 & Acacia senegal & Senegal & de Lajudie et al. (1998) \\
\hline \multicolumn{4}{|c|}{ Mesorhizobium loti } \\
\hline LMG $6125^{\mathrm{T}}$ & Lotus corniculatus & New Zealand & LMG \\
\hline \multicolumn{4}{|c|}{ Sinorhizobium saheli } \\
\hline ORS $609^{\mathrm{T}}$ & Sesbania canabina & Senegal & de Lajudie et al. (1994) \\
\hline ORS 600 & Sesbania pachycarpa & Senegal & de Lajudie et al. (1994) \\
\hline \multicolumn{4}{|c|}{ Sinorhizobium terangae } \\
\hline ORS 51 & Sesbania rostrata & Senegal & de Lajudie et al. (1994) \\
\hline ORS 15 & Sesbania sp. & Senegal & de Lajudie et al. (1994) \\
\hline ORS $1009^{\mathrm{T}}$ & Acacia laeta & Senegal & de Lajudie et al. (1994) \\
\hline ORS 1016 & Acacia laeta & Senegal & de Lajudie et al. (1994) \\
\hline LMG 6463 & Sesbania rostrata & Senegal & de Lajudie et al. (1994) \\
\hline \multicolumn{4}{|c|}{ Sinorhizobium meliloti } \\
\hline LMG $6133^{\mathrm{T}}$ & Medicago sativa & Virginia, USA & LMG \\
\hline LMG 6130 & Medicago sativa & Australia & LMG \\
\hline \multicolumn{4}{|c|}{ Agrobacterium biovar 2} \\
\hline \multicolumn{4}{|c|}{ Agrobacterium rubi } \\
\hline LMG $156^{\mathrm{T}}$ & Rubus ursinus & USA, 1942 & \\
\hline \multicolumn{4}{|c|}{ Agrobacterium biovar 1} \\
\hline LMG 196 & crown gall & USA & Kersters et al. (1973) \\
\hline \multicolumn{4}{|c|}{ Agrobacterium vitis } \\
\hline LMG 257 & Vitis vinifera & Crete, Greece & \\
\hline
\end{tabular}

* Grouping according to ITS-PCR-RFLP.

PCR/RFLP of 16S rRNA genes. A bacterial colony was homogenized vigorously into $100 \mu \mathrm{l}$ of $0 \cdot 1 \%$ Tween 20 solution. The cell suspension was heated at $95-100{ }^{\circ} \mathrm{C}$ for $10 \mathrm{~min}$ and was directly used for PCR assay. DNA was amplified by mixing $3 \mu \mathrm{l}$ of the suspension with the 16S PCR buffer $(10 \mathrm{mM}$ Tris $/ \mathrm{HCl}, \mathrm{pH} 8.3 ; 50 \mathrm{mM} \mathrm{KCl} ; 0.01 \%$ gelatin), $2 \mu \mathrm{l}$ each of the $10 \mathrm{mM}$ dNTPs (dATP, dCTP, dGTP, dTTP), $1.5 \mu 1100 \mathrm{mM} \mathrm{MgCl}_{2}, 1 \mu$ each primer fD1 and rD1 (Weisburg et al., 1991) which correspond to positions 8-27 and $1524-1540$ respectively, of the E. coli $16 \mathrm{~S}$ rRNA gene and $80 \cdot 2 \mu 1$ sterilized water. The mixture was sealed with a thin layer of paraffin oil and heated for $3 \mathrm{~min}$ at $95^{\circ} \mathrm{C}$. A $2.5 \mu \mathrm{l}$ aliquot of diluted Taq polymerase in Extender and deionized water $(1: 1: 3$, by vol.) was added to the mixture. The PCR amplification was carried out in a $100 \mu \mathrm{l}$ reaction volume and performed with a thermal reactor HYBAID. The PCR temperature profile was as follows: initial denaturation at $94{ }^{\circ} \mathrm{C}$ for $3 \mathrm{~min} ; 35$ amplification cycles (denaturation $94^{\circ} \mathrm{C}$ for $1 \mathrm{~min}$, primer annealing $55^{\circ} \mathrm{C}$ for $1 \mathrm{~min}$ and extension $72^{\circ} \mathrm{C}$ for $2 \mathrm{~min}$ ); final extension at $72{ }^{\circ} \mathrm{C}$ for $3 \mathrm{~min}$.
An excess of restriction enzymes ( $5 \mathrm{U} /$ reaction) was used to digest a $10 \mu \mathrm{l}$ aliquot of PCR product. Restricted DNAs with HhaI, HinfI, MspI and Sau3AI were analysed by horizontal agarose $(3 \%, \mathrm{w} / \mathrm{v})$ gel electrophoresis carried out at $80 \mathrm{~V}$ for $3.5 \mathrm{~h}$. Gels were stained in an aqueous solution of ethidium bromide $\left(1 \mathrm{mg} \mathrm{ml}^{-1}\right)$ and photographed under UV illumination using a Polaroid 55 positive/negative film.

Auxanographic tests. API galleries (API 50CH, API 50AO and API 50AA; bioMérieux) were used to test the assimilation of 147 organic compounds as sole carbon sources (Kersters et al., 1984). Inocula were obtained from $36 \mathrm{~h}$ YMA slant cultures. After incubation of the galleries for 1, 2,4 and $7 \mathrm{~d}$ at $30^{\circ} \mathrm{C}$, growth of the strains was checked and scored as described previously (Kersters et al., 1984). The levels of interstrain similarity $(S)$ were calculated by using a similarity distance coefficient derived from the Canberra metric coefficient (dCanb) (Sneath \& Sokal, 1973) as follows: $S=100 \times(1-\mathrm{dCanb})$.

Plant nodulation tests. Phaseolus vulgaris, Vigna unguiculata and Glycine max seeds were surface sterilized successively 
Table 2. Allele profiles at 12 enzyme loci forming 13 ETs among reference strains of $R$. etli and $R$. tropici and Rhizobium strains isolated from Phaseolus vulgaris in West Africa

\begin{tabular}{|c|c|c|c|c|c|c|c|c|c|c|c|c|c|}
\hline \multirow[t]{2}{*}{ ET } & \multirow[t]{2}{*}{ Strain } & \multicolumn{12}{|c|}{ Alleles at the following enzyme loci* } \\
\hline & & IDH & MDH & G6P & XDH & HEX & GD2 & $\mathrm{ACO}$ & ALA & PGM & IPO & EST1 & EST2 \\
\hline 1 & ISRA 27, ISRA 59 & 7 & 5 & 4 & 6 & 7 & 7 & 7 & 4 & 7 & 5 & 4 & 5 \\
\hline 2 & ISRA 61 & 7 & 5 & 5 & 6 & 7 & 7 & $7 \cdot 2$ & 4 & 7 & 5 & 4 & 5 \\
\hline 3 & ISRA 69 & 7 & 5 & 4 & 6 & 7 & 7 & $7 \cdot 2$ & 4 & 7 & 5 & $3 \cdot 5$ & 5 \\
\hline 4 & Groups II.1 \& II.2 strains & 5 & 5 & 5 & 5 & 5 & 5 & 5 & 3 & 5 & 5 & 3 & 5 \\
\hline 5 & Group I strains & 5 & 7 & 3 & $6 \cdot 5$ & 5 & 5 & 5 & 3 & 5 & 7 & 6 & 8 \\
\hline 6 & R. etli BRA 5 & 5 & 5 & 5 & 6 & 5 & 5 & 5 & 3 & 5 & 5 & 3 & 5 \\
\hline 7 & R. etli $\mathrm{CFN} 42^{\mathrm{T}}$ & 5 & 5 & 5 & 5 & 5 & 5 & 5 & 3 & 5 & 5 & $4 \cdot 5$ & 5 \\
\hline 8 & R. tropici type A CFN $299^{\mathrm{T}}$ & 5 & 6 & 3 & 6 & 5 & 5 & 5 & 3 & 5 & 3 & 6 & $6 \cdot 5$ \\
\hline 9 & R. tropici type B CIAT $899^{\mathrm{T}}$ & 5 & 7 & 3 & $6 \cdot 5$ & 5 & 5 & 5 & 3 & 5 & 5 & 6 & 8 \\
\hline 10 & R. etli $\mathrm{F} 6$ & 5 & 5 & 4 & 6 & 5 & 5 & 5 & 3 & 5 & 5 & 4 & 5 \\
\hline 11 & R. etli $\mathrm{F} 8$ & 7 & 5 & 5 & 6 & 7 & 7 & 7 & 4 & 7 & 5 & 4 & 5 \\
\hline 12 & R. etli $\mathrm{F} 16$ & 7 & 5 & 4 & $5 \cdot 5$ & 7 & 7 & 7 & 4 & 7 & 5 & 3 & 5 \\
\hline 13 & R. etli Viking 1 & 5 & 5 & 3 & 6 & 5 & 5 & 5 & 3 & 5 & 5 & $5 \cdot 5$ & $5 \cdot 5$ \\
\hline
\end{tabular}

* IDH, Isocitrate dehydrogenase; $\mathrm{MDH}$, malate dehydrogenase; G6P, glucose-6-phosphate dehydrogenase; XDH, xanthine dehydrogenase; HEX, hexokinase; GD2, glutamate dehydrogenase; ACO, aconitase; ALA, alanine dehydrogenase; PGM, phosphoglucomutase; IPO, indophenol oxidase; and EST, esterases.

Table 3. Groupings of ISRA Rhizobium strains nodulating Phaseolus vulgaris using PCR-RFLP analysis of the 16S-23S RNA intergenic spacer

-, DNA not digested with the corresponding enzyme.

\begin{tabular}{|lcccccccc|}
\hline $\begin{array}{l}\text { Group or subgroup } \\
\text { of strains }\end{array}$ & \multicolumn{7}{c|}{ Restriction pattern of amplified ITS gene digested with: } \\
\cline { 2 - 9 } & AluI & DdeI & HhaI & HinfI & MspI & PalI & RsaI & TaqI \\
\hline Group I & A1 & D1 & Hh1 & $\mathrm{Hi}^{-}$ & M1 & P1 & $\mathrm{R} 1$ & $\mathrm{~T} 1$ \\
Subgroup II.1 & A2 & D2 & Hh2 & $\mathrm{Hi}^{-}$ & M2 & P2 & $\mathrm{R}^{-}$ & T2 \\
Subgroup II.2 & A2 & D2 & Hh2 & $\mathrm{Hi}^{-}$ & M2 & P2 & $\mathrm{R}^{-}$ & T2 \\
Subgroup II.3 & A4 & D4 & Hh4 & $\mathrm{Hi}^{-}$ & M4 & P4 & $\mathrm{R}^{-}$ & T4 \\
\hline
\end{tabular}

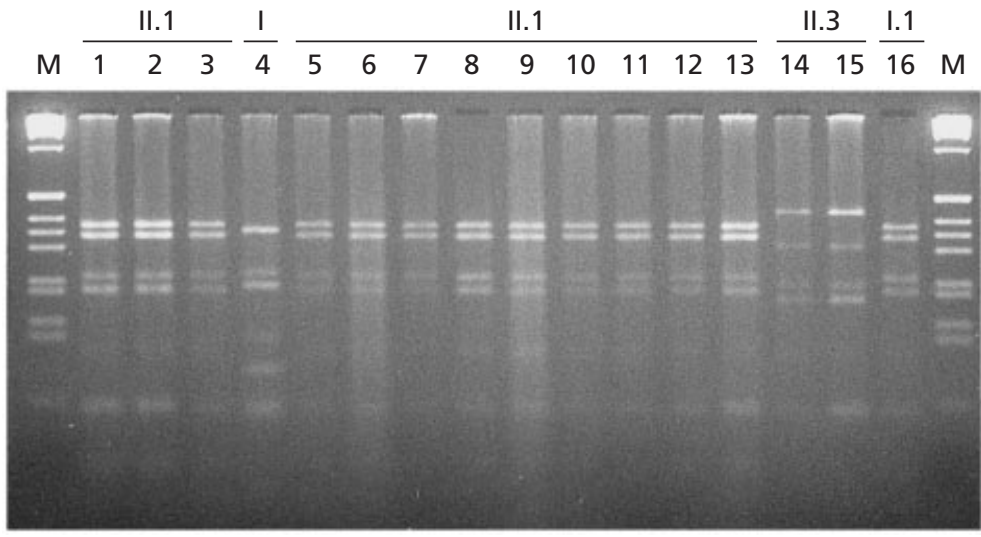

Fig. 1. Restriction patterns of $P C R$-amplified fragment of 16S-23S rDNA digested with Taql. Lanes: M, molecular mass marker; (subgroup II.1) 1, ISRA 351; 2, ISRA 355; 3, ISRA 553; 5, ISRA 556; 6, ISRA 558; 7, ISRA $562 ; 8$, ISRA 563; 9, ISRA 567; 10, ISRA 570; 11, ISRA 576; 12, ISRA 586; 13, ISRA 77; 16, ISRA 319; Group I; 4, ISRA 554; (subgroup II.3) 14, ISRA 27; 15, ISRA 59.

with $95 \%(\mathrm{v} / \mathrm{v})$ ethanol for $3 \mathrm{~min}$ and $0 \cdot 1 \% \mathrm{HgCl}_{2}$ for $3 \mathrm{~min}$ followed by several washes in sterile distilled water. Acacia senegal, Leucaena leucocephala, Acacia tortilis subsp. raddiana, Faidherbia albida, Sesbania rostrata and Acacia seyal seeds were scarified and surface-sterilized with concen- trated sulfuric acid for $14,20,25,30,45$ and $60 \mathrm{~min}$, respectively. After treatment, the seeds were washed several times with water to eliminate any trace of acid and $\mathrm{HgCl}_{2}$. The seeds were first allowed to germinate in sterile Petri dishes containing $0.8 \%$ water agar for $24-48 \mathrm{~h}$, then 
Table 4. Number of alleles and genetic diversity $(h)$ at the loci encoding the 12 enzymes in ETs observed in the Rhizobium strains ISRA isolated from Phaseolus vulgaris and the reference strains

$h=1-\Sigma \mathrm{x}_{\mathrm{i}}{ }^{2}(n /(n-1))$ where $x_{\mathrm{i}}$ is the frequency of the $i$ th allele and $n$ is the number of ETs.

\begin{tabular}{|c|c|c|c|c|}
\hline \multirow{2}{*}{$\begin{array}{l}\text { Enzyme } \\
\text { locus* }\end{array}$} & \multicolumn{2}{|c|}{ Thirteen ETs $\dagger$} & \multicolumn{2}{|c|}{ Four ETst } \\
\hline & $\begin{array}{l}\text { No. } \\
\text { alleles }\end{array}$ & $\boldsymbol{h}$ & $\begin{array}{l}\text { No. } \\
\text { alleles }\end{array}$ & $\boldsymbol{h}$ \\
\hline IDH & 2 & $0 \cdot 513$ & 2 & $0 \cdot 499$ \\
\hline MDH & 3 & $0 \cdot 011$ & 1 & $0 \cdot 000$ \\
\hline G6P & 3 & $0 \cdot 717$ & 2 & 0.667 \\
\hline $\mathrm{XDH}$ & 3 & $0 \cdot 616$ & 2 & 0.499 \\
\hline HEX & 2 & 0.513 & 2 & 0.499 \\
\hline GD2 & 2 & $0 \cdot 513$ & 2 & $0 \cdot 499$ \\
\hline $\mathrm{ACO}$ & 3 & 0.590 & 3 & $0 \cdot 832$ \\
\hline ALA & 2 & $0 \cdot 513$ & 2 & 0.499 \\
\hline PGM & 2 & $0 \cdot 513$ & 2 & 0.499 \\
\hline IPO & 3 & $0 \cdot 295$ & 1 & 0.000 \\
\hline EST1 & 5 & $0 \cdot 846$ & 3 & 0.832 \\
\hline EST2 & 4 & 0.527 & 1 & $0 \cdot 000$ \\
\hline Mean & $2 \cdot 83$ & 0.547 & 1.91 & $0 \cdot 444$ \\
\hline
\end{tabular}

* For abbreviations of enzyme loci see the footnote to Table 2.

$\dagger$ The 13 ETs of ISRA and reference strains.

t The four ETs of ISRA $R$. etli strains.

transferred to tubes containing Jensen slant agar (Vincent, 1970) for root nodulation tests (6-8 plants were routinely tested with each strain). All 58 new isolates were tested for their ability to nodulate these plants.

\section{RESULTS AND DISCUSSION}

\section{Collection of strains}

Fifty-eight rhizobial isolates (Table 1) were purified from root nodules of common bean and included in the Senegalese Institute of Agricultural Research (ISRA)/Microbiological Resources Center (MIRCEN) culture collection. These isolates originate from different geographical and ecological areas in Senegal and Gambia, including fields in the Niayes zone where common bean is mainly cultivated in Senegal, and were obtained either by direct isolation from naturally occurring nodules or by trapping Rhizobium on sterile young plants inoculated with collected soil samples.

\section{Morphological and physiological characteristics}

All isolates were fast growers and, based on their morphological and physiological characteristics, we defined two groups (I and II) among them. In YM liquid medium, strains ISRA 350, 352, 354 and 554 (forming the so-called group I) grew at temperatures up to $44^{\circ} \mathrm{C}$ whereas the maximum growth temperature of the 54 other isolates (forming the so-called group II) was $40{ }^{\circ} \mathrm{C}$. On PY medium, the texture of colonies of group I strains was creamy whereas colonies of group II had a gummy texture. Strains of group I were able to grow on LB medium whereas strains of group II could not grow on this medium. These morphological and physiological characteristics of group I strains such as the ability to grow on LB medium and colony morphology on PY medium as well as their maximal growth temperature matched characteristics reported for R. tropici (Martínez-Romero et al., 1991). On the other hand, group II strains shared characteristics of R. etli (Segovia et al., 1993).

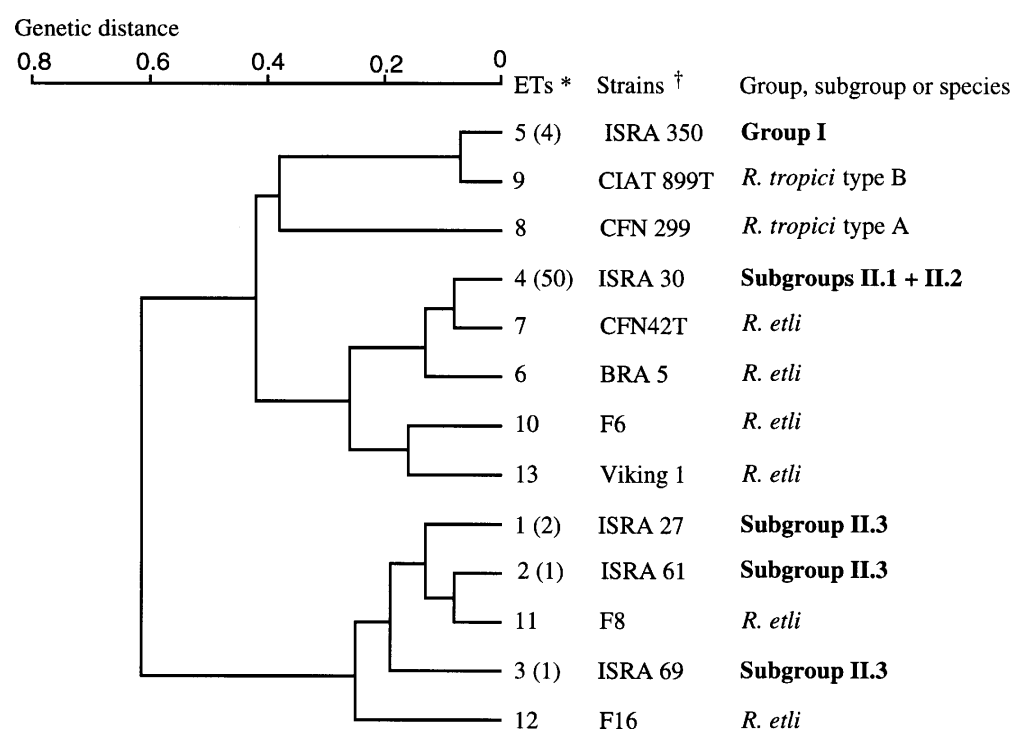

Fig. 2. Similarity among 13 ETs of ISRA Rhizobium strains isolated from common bean (Phaseolus vulgaris) and reference strains based on electrophoretically detectable allele variation at 12 enzyme loci. *Values in parentheses correspond to the number of strains in each ET. †Only one strain was represented for each ET. 


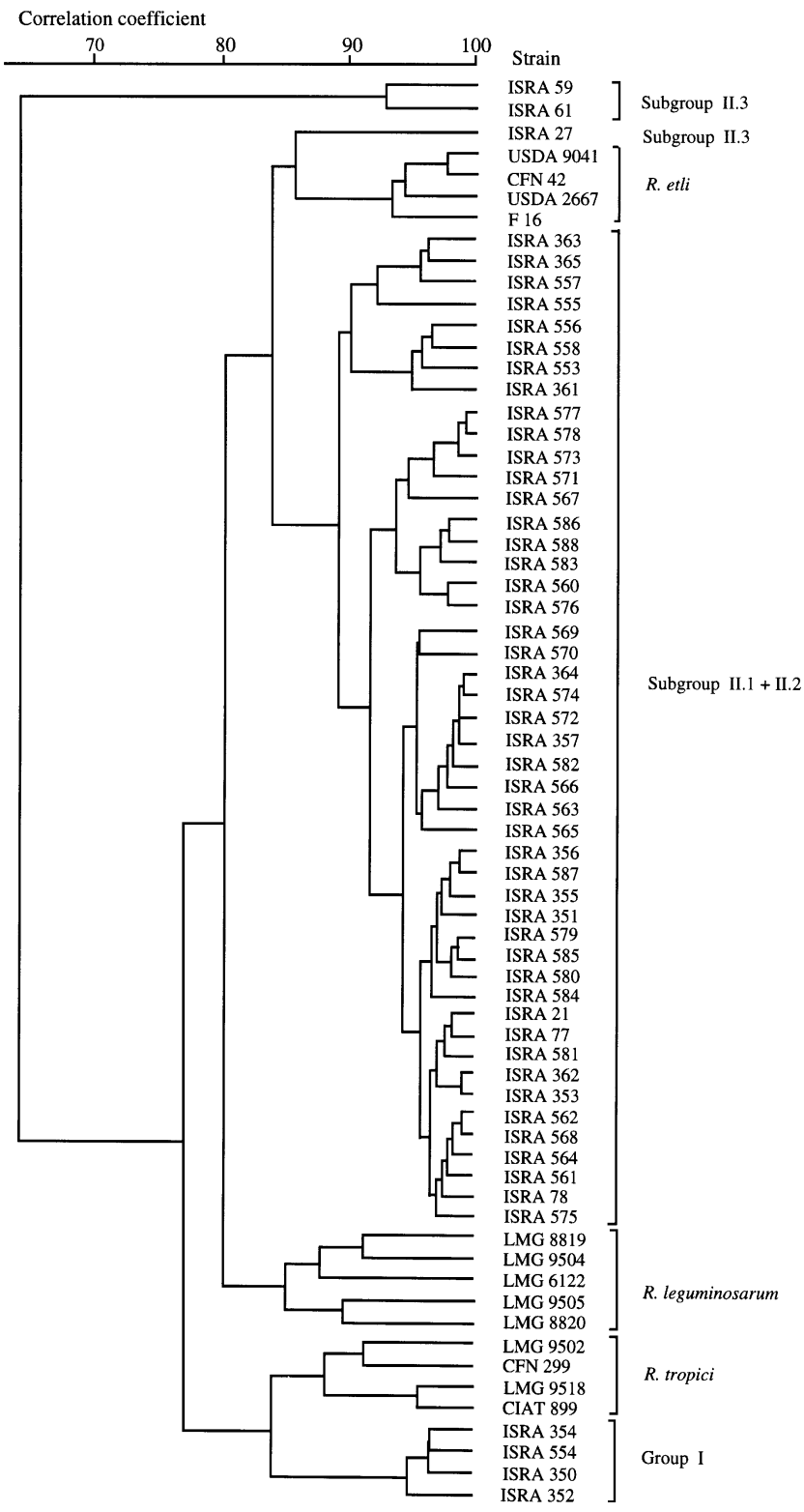

Fig. 3. Dendrogram showing the similarity among the electrophoretic protein patterns (SDS-PAGE) of ISRA and reference strains based on the mean correlation coefficient values which were grouped by the unweighted pair group method with averages.

\section{PCR/RFLP of 16S-23S ITS region}

Analysis of the PCR product of the 16S-23S ITS DNA region was reported as a useful method to evidence diversity among bacterial populations even at the intraspecific level (Barry et al., 1991; Jensen et al., 1993; Laguerre et al., 1996). The PCR amplified $16 \mathrm{~S}-23 \mathrm{~S}$ rDNA intergenic spacer regions of the 58 new isolates were digested with eight restriction enzymes, leading to polymorphic patterns (Table 3, Fig. 1). Four different groups of strains were identified. One corresponded to the four group I strains which shared the same restriction pattern type. Group II strains formed a separate type with three subgroups, one major (subgroup II.1, 45 strains, see Table 1), and two minor (subgroups II.2 and II.3). The only difference between subgroups II.1 and II.2 was that DNA of the former could not be digested by HinfI. More important were the differences exhibited by the four strains of subgroup II.3 (see Table 1) showing a separate profile. RsaI restricted only ITS amplificates of strains from group I.

\section{MLEE}

The genetic relationships between the 58 isolates and reference strains of Rhizobium tropici and Rhizobium etli were further examined by performing MLEE, a technique previously employed in taxonomic studies of rhizobia that nodulate bean plants (Piñero et al., 1988; Martínez-Romero et al., 1991; Segovia et al., 1991; Souza et al., 1994). The twelve enzyme loci analysed were polymorphic with a number of alleles ranging from two to five electromorphs. The mean number of alleles was 2.83 (Table 4). There were 13 distinctive multilocus genotypes or ETs as indicated in Table 2. The electrophoretic mobilities at the twelve enzyme loci showed five ETs among the 58 isolates. The genetic diversity at the enzyme locus for the four ETs of the 54 ISRA strains of group II was 0.44 (Table 4). On the basis of the analysis of the genetic distance, the rhizobial strains nodulating bean in Senegal and Gambia are clustered into two groups. The four strains of group I (ISRA 350, ISRA 352, ISRA 354 and ISRA 554 ) corresponded to ET 5 and grouped with $R$. tropici type B. Group II strains exhibited four ETs, different from, but all similar to, those of $R$. etli. The majority of group II strains (the 50 strains of subgroup II.1 and II.2) have a single ET 4, highly similar to that of the type strain of $R$. etli $\mathrm{CFN} 42^{\mathrm{T}}$. The four remaining strains of group II (belonging to subgroup II.3) corresponded to ET 1 (ISRA 27 and ISRA 59), ET 2 (ISRA 61) and ET 3 (ISRA 69), all similar to other $R$. etli reference strains (Table 2, Fig. 2). Both group II and $R$. etli reference strains showed ET heterogeneity among them but their ETs all clustered together.

Nour et al. (1994) reported good agreement of results obtained by RFLP analysis of the rDNA $16 \mathrm{~S}-23 \mathrm{~S}$ intergenic spacer and MLEE for rhizobia nodulating chickpea (Cicer arietinum). Here both methods confirmed on the whole the distinction of the two main groups I and II among the new isolates, similar to $R$. etli and $R$. tropici type $\mathrm{B}$, but with different subgroupings : three ETs were found among subgroup II.3 of ITS-PCR-RFLP, while two ITS-PCR-RFLP subgroups were distinguished among the single ET 4.

\section{SDS-PAGE of total bacterial proteins}

The SDS-PAGE whole-cell protein patterns of 54 of the rhizobial isolates from Senegal were scanned and numerically analysed, together with those of reference strains available in our database and representing Rhizobium species nodulating bean plants, $R$. tropici, 


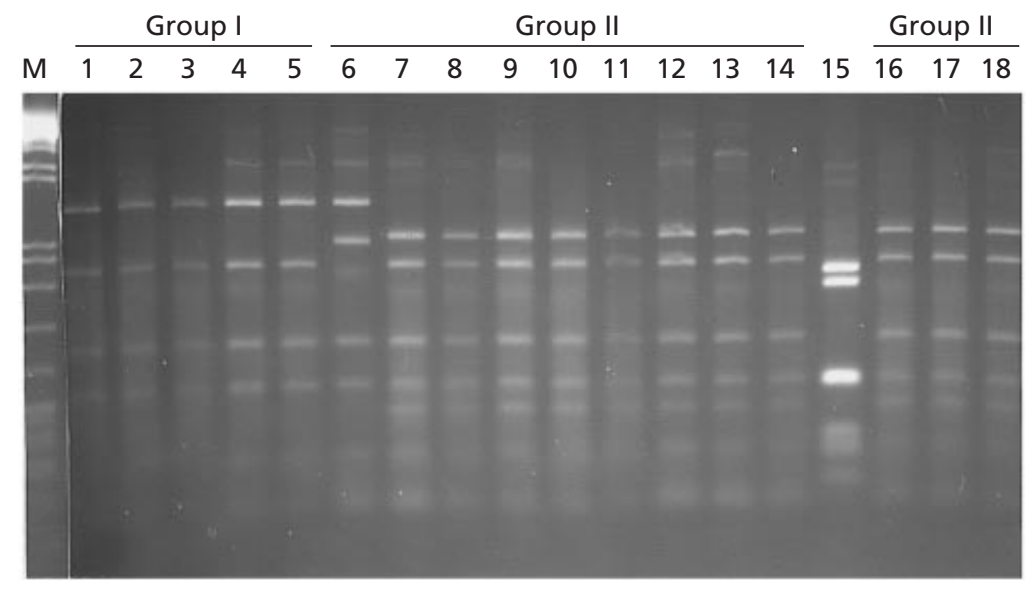

Fig. 4. Restriction patterns of $P C R$-amplified fragment of $16 \mathrm{~S}$ rRNA genes digested with Mspl. Lanes: M, molecular mass marker; 1 , ISRA $350 ; 2$, ISRA $352 ; 4$, ISRA 354; 5, ISRA 554 ; reference strains $3, R$. tropici type $B$ CIAT 899; 6, R. tropici type A CFN 299; 7, ISRA $27 ; 8$, ISRA 59; 9, ISRA 61; 10, ISRA 69; 12, ISRA 30; 13, ISRA 77; 14, ISRA 353; 16, ISRA 566; 17, ISRA 577; 18, ISRA 584; reference strain $11, R$. etli CFN $42 ; 15$, $R$. leguminosarum USDA 2370. Group I, restriction pattern types of $R$. tropici type $\mathrm{B}_{i}$ group II, restriction pattern types of $R$. etli.

$R$. etli and $R$. leguminosarum. Bean isolates from Senegal and Gambia essentially grouped in two separate protein gel electrophoretic clusters. The results are presented as a similarity dendrogram in Fig. 3. The four isolates of group I clustered together at a mean correlation coefficient $(r)$ of $94 \%$. The majority of group II strains formed a second cluster at a mean correlation coefficient of $89 \%$. Group I and group II were respectively related to reference strains of $R$. tropici and $R$. etli at a moderate mean coefficient correlation of $83.5 \%$. Strain ISRA 27 (belonging to subgroup II.3) grouped separately from the core of other isolates of group II, closer to R. etti. Two other strains of subgroup II.3, ISRA 59 and ISRA 61, grouped together, outside the big groups I and II. The fourth strain of subgroup II.3, ISRA 69, was not included in this study.

\section{PCR-RFLP of 16S rRNA genes}

PCR-RFLP analysis of 16S rRNA coding gene has been reported to be useful in Rhizobium taxonomy since results obtained are in good agreement with those from sequence analysis of the 16S rRNA coding gene and DNA-DNA hybridization (Laguerre et al., 1993). Four restriction endonucleases HhaI, Hinf $\mathrm{I}$, $M s p$ I and Sau3AI were used to digest 16S rRNA gene PCR amplificates of a selection of 29 Senegalese isolates, including all strains of group I, subgroup II.2 and II.3 (Table 1), and 16 representative strains of subgroup II.1 (ISRA 30, ISRA 353, ISRA 355, ISRA 580, ISRA 576, ISRA 584, ISRA 555, ISRA 577, ISRA 562, ISRA 566, ISRA 567, ISRA 570, ISRA 571, ISRA 578, ISRA 581, ISRA 584). The restriction patterns generated by HhaI, HinfI and Sau3AI were similar for all isolates, but two distinct restriction patterns were detected with $M s p I$ among our isolates (Fig. 4): the first was exhibited by group I strains and R. tropici CIAT $899^{\mathrm{T}}$; the second pattern was exhibited by group II strains and $R$. etli CFN $42^{\mathrm{T}}$. $R$. tropici strains, genomically described by sequence of their $16 \mathrm{~S}$ rRNA gene, DNA-DNA hybridization and rDNA organization, were separated into two subgroups, namely type A and type B (Martínez-Romero et al.,

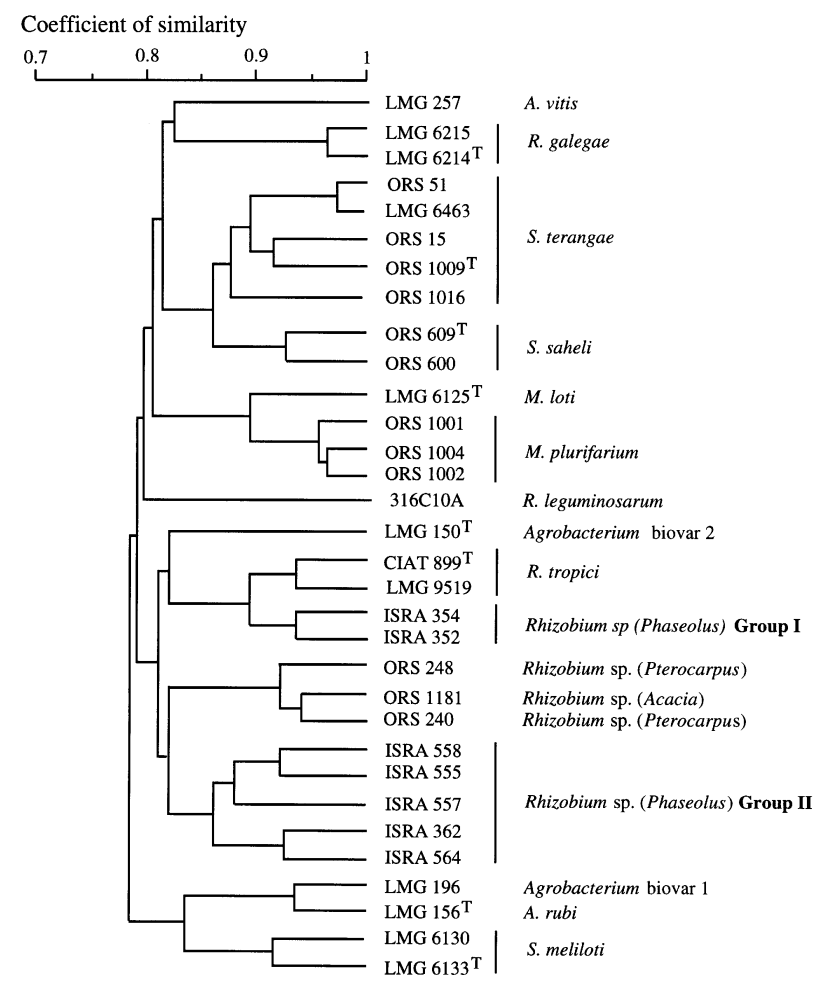

Fig. 5. Dendrogram showing the differences of auxanographic characteristics among ISRA strains and reference strains, obtained from an unweighted pair group method with averages cluster analysis of Canberra metric similarity coefficients based on 147 auxanographic characteristics.

1991; Geniaux et al., 1993). Restriction patterns of $R$. tropici type A and of $R$. tropici type B were different, and the pattern of group I rhizobial strains was related to that of the latter.

\section{Analysis of auxanographic results}

Only the first acquired isolates were tested for assimilation of 147 organic compounds as sole carbon sources, using the API 50 system. Group I was represented by strains ISRA 352, ISRA 354 and group 


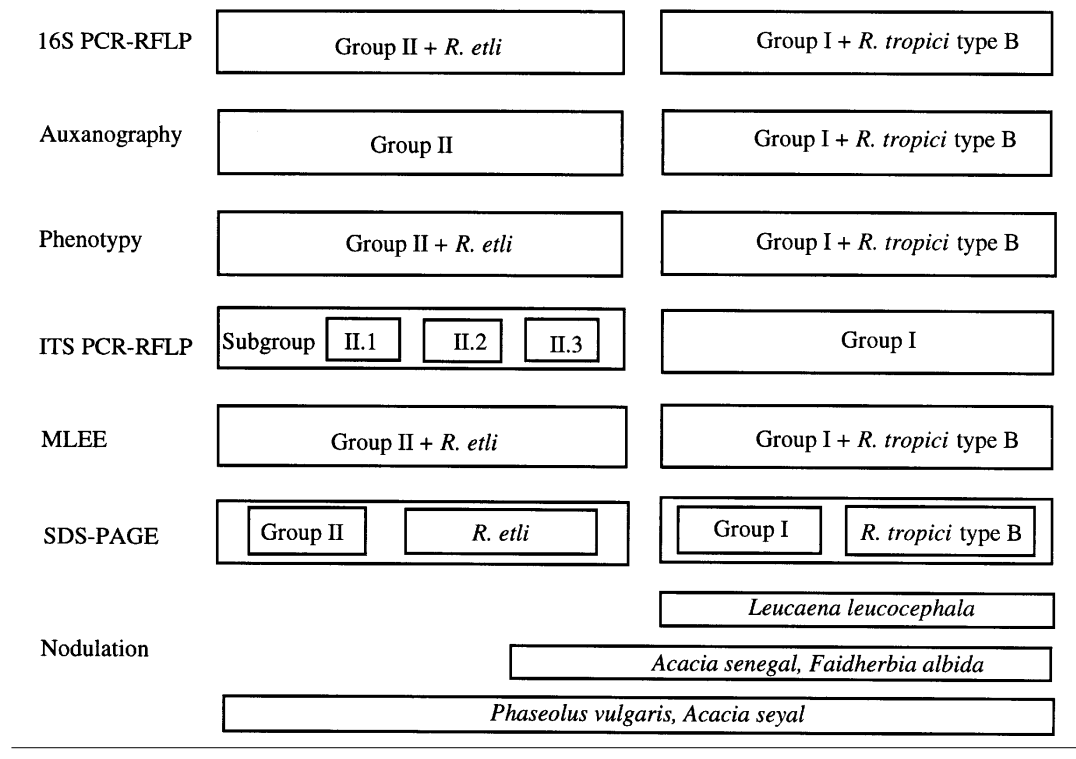

Fig. 6. Diagram summarizing results of polyphasic characterization of beannodulating rhizobia in Senegal and Gambia.
II was represented by ISRA 555, ISRA 557, ISRA 558, ISRA 564 and ISRA 362. The reproducibility of the test was good. The mean interstrain similarity values for strains tested in duplicate were between 88 and $92 \%$. Results for representative strains of several Rhizobium species and related groups (including representatives of the Agrobacterium biovars, but not $R$. etli) were available in the database of our research group and were included in the numerical analysis. The results are shown as a limited dendrogram in Fig. 5. Group I formed a common cluster with $R$. tropici (similarity coefficient of $89 \%$ ). At a similarity coefficient of $86 \%$, cluster II could be distinguished as a separate cluster.

\section{Host specificity}

Bean nodulating rhizobia have been reported to have a broad host range (Hernandez-Lucas et al., 1995; Geniaux et al., 1993; Amarger et al., 1997). The 58 new nodule isolates could all nodulate their original host plant, Phaseolus vulgaris, and Acacia seyal whereas none of them nodulated Glycine max and Sesbania rostrata. Some differences in host ranges were found among the isolates. Only strains of group I were able to effectively nodulate Leucaena leucocephala, similarly to R. tropici (Martínez-Romero et al., 1991). The four strains of group I and the four strains of subgroup II.1 (ISRA 27, ISRA 59, ISRA 61 and ISRA 69) nodulated Faidherbia albida and Acacia senegal.

By all the criteria tested and results summarized in Fig. 6, group I African isolates were $R$. tropici type B strains while group II corresponded to $R$. etli.

Population diversity studies of bean nodulating rhizobia from areas where this plant is native and extensively grown have shown a large genetic diversity. This has been observed both for $R$. tropici from South American soils (Martínez-Romero et al., 1991) and for R. etli in Mesoamerica (Piñero et al., 1988; Segovia et al., 1991; Eardly et al., 1995). For both species a large number of ETs have been obtained with $H$ values (diversity index) of 0.363 for $R$. tropici type $\mathrm{B}$ strains and larger than 0.5 for $R$. etli. In comparison we report here only one ET for the $R$. tropici strains in contrast to the 18 ETs for the type B $R$. tropici strains analysed previously (Martínez-Romero et al., 1991). Similarly, only four ETs were obtained for our $R$. etli strains while a maximum of 70 ETs was reported in $R$. etli isolated from a limited geographical area in Mexico (Caballero-Mellado \& Martínez-Romero, 1999). Also indicative of the restricted genetic diversity of the bean rhizobia population in Africa is the large dominance of a single genotype: 50 strains have a single ET. The limited genetic diversity encountered in Senegal and Gambia may be related to the fact that bean is an introduced crop in Africa. It is remarkable that in West, East and South Africa, bean is naturally nodulated by the same species of rhizobia as those nodulating bean at its site of origin. It has been supposed that bean symbiotic rhizobia are cosmopolitan. An alternative hypothesis was suggested by Sessitsch et al. (1997) to explain the presence of $R$. etli in Austrian soil. They proposed that the rhizobia was co-introduced with bean seeds. The presence of $R$. etli on Phaseolus vulgaris seeds was demonstrated, and the rhizobia remain viable on the seeds for years (PérezRamírez et al., 1998). We may suppose that $R$. tropici could be also transported on bean seeds harvested from South America where $R$. tropici is native. The existence of Rhizobium on seeds may explain the presence of $R$. etli in Africa and the limited genetic diversity encountered may be related to the founder principle, with only a few clones (founders) migrating and surviving at the introduced place.

Africa has been proposed to be the site of origin of the Leguminosae family (Raven \& Polhill, 1981), and as such a large diversity of legume species and their symbionts exist. If the symbionts of Phaseolus vulgaris 
were originally from Africa, we should recover the largest diversity there, but that does not seem to be the case. It seems more probable that bean symbionts coevolved with their host at the site where they diverged and prospered.

\section{ACKNOWLEDGEMENTS}

We thank bioMérieux, Montalieu-Vercieu, France, for supplying API galleries. This work was partially supported by UNESCO grant SC/RP/206.575.6. We are grateful to J. Bakhoum, ORSTOM, Senegal, and M. A. Rogel, CIFN/ UNAM, Mexico, for technical assistance. M. G. is indebted to the Fund for Scientific Research-Flanders (Belgium), for research and personnel grants.

\section{REFERENCES}

Amarger, N., Macheret, V. \& Laguerre, G. (1997). Rhizobium gallicum sp. nov. and Rhizobium giardinii sp. nov., from Phaseolus vulgaris nodules. Int J Syst Bacteriol 47, 996-1006.

Anyango, B., Wilson, K. J., Beynon, J. L. \& Giller, K. E. (1995). Diversity of Rhizobium nodulating Phaseolus vulgaris L. in two Kenyan soils with contrasting pHs. Appl Environ Microbiol 61, 4016-4021.

Barry, T., Colleran, G., Glennon, M., Ducican, L. K. \& Gannon, F. (1991). The $16 \mathrm{~S} / 23 \mathrm{~S}$ ribosomal spacer region as a target for DNA probes identity eubacteria. PCR Methods Applic 1, 51-56. van Berkum, P., Beyene, B. \& Eardly, B. D. (1996). Phylogenetic relationships among Rhizobium species nodulating the common bean (Phaseolus vulgaris L.). Int J Syst Bacteriol 46, 240-244.

Boucher, C. A., Van Gijsegem, F., Barberis, P., Arlat, A. M. \& Zischek, C. (1987). Pseudomonas solanacearum genes controlling both pathogenicity on tomato and hypersensitivity on tobacco are clustered. J Bacteriol 169, 5626-5632.

Caballero-Mellado, J. \& Martínez-Romero, E. (1999). Soil fertilization limits the genetic diversity of Rhizobium in bean nodules. Symbiosis 26, 111-121.

Dagutat, H. \& Steyn, P. L. (1995). Taxonomy and distribution of rhizobia indigenous to South African soils. In Nitrogen Fixation: Fundamentals and Applications, pp. 683-686. Edited by I. A. Tikhonovich, N. A. Provorov, V. I. Romanov \& W. E. Newton. Boston: Kluwer.

Eardly, B. D., Wang, F., Whittam, T. S. \& Selander, R. K. (1995). Species limits in Rhizobium populations that nodulate the common bean (Phaseolus vulgaris). Appl Environ Microbiol 61, 507-512.

Geniaux, E., Laguerre, G. \& Amarger, N. (1993). Comparison of geographically distant populations of Rhizobium isolated from root nodules of Phaseolus vulgaris. Mol Ecol 2, 295-302.

Geniaux, E., Flores, M., Palacios, R. \& Martínez, E. (1995). Presence of megaplasmids in Rhizobium tropici and further evidence of differences between the two Rhizobium tropici subtypes. Int $J$ Syst Bacteriol 45, 392-394.

Hernandez-Lucas, I., Segovia, L., Martínez-Romero, E. \& Pueppke, S. G. (1995). Phylogenetic relationships and host range of Rhizobium spp. that nodulate Phaseolus vulgaris L. Appl Environ Microbiol 61, 2775-2779.

Jensen, M. A., Webster, J. A. \& Straus, N. (1993). Rapid identification of bacteria on the basis of polymerase chain reactionamplification ribosomal DNA spacer polymorphisms. Appl Environ Microbiol 59, 945-952.
Jordan, D. C. (1984). Family III Rhizobiaceae Conn 1938. In Bergey's Manual of Systematic Bacteriology, vol. 1, pp. 234-245. Edited by N. R. Krieg \& J. G. Holt. Baltimore: Williams \& Wilkins.

Kersters, K., De Ley, J., Sneath, P. H. A. \& Sackin, M. (1973). Numerical taxonomic analysis of Agrobacterium. $J$ Gen Microbiol 78, 227-239.

Kersters, K., Hinz, K. H., Hertle, A., Segers, P., Lievens, A., Siegmann, O. \& De Ley, J. (1984). Bordetella avium sp. nov., isolated from the respiratory tracts of turkeys and other birds. Int J Syst Bacteriol 34, 56-70.

Kiredjian, M., Holmes, B., Kersters, K., Guilvout, J. \& De Ley, J. (1986). Alcaligenes piechaudii, a new species from human clinical specimens and the environment. Int $J$ Syst Bacteriol 36, 282-287.

Laemmli, U. K. (1970). Cleavage of structural proteins during the assembly of the head of bacteriophage T4. Nature 227, 680-685.

Laguerre, G., Fernandez, M. P., Edel, V., Normand, P. \& Amarger, N. (1993). Genomic heterogeneity among French Rhizobium strains isolated from Phaseolus vulgaris L. Int J Syst Bacteriol 43, 761-767.

Laguerre, G., Mavingui, P., Allard, M. R., Charnay, M. P., Louvrier, P., Mazurier, S. I., Rigottier-Gois, L. \& Amarger, N. (1996). Typing of rhizobia by PCR DNA fingerprinting and PCR-restriction fragment length polymorphism analysis of chromosomal and symbiotic gene regions: application to Rhizobium leguminosarum and its different biovars. Appl Environ Microbiol 62, 2029-2036.

de Lajudie, P., Willems, A., Pot, B. \& 7 other authors (1994). Polyphasic taxonomy of rhizobia: Emendation of the genus Sinorhizobium and description of Sinorhizobium saheli sp. nov. and Sinorhizobium teranga sp. nov. Int J Syst Bacteriol 44, 715-733.

de Lajudie, P., Willems, A., Nick, G. \& 9 other authors (1998). Characterization of tropical tree rhizobia and description of Mesorhizobium plurifarium sp. nov. Int J Syst Bacteriol 48, 369-382.

Martínez, E., Pardo, M. A., Palacios, R. \& Cevallos, M. A. (1985). Reiteration of nitrogen fixation gene sequences and specificity of Rhizobium in nodulation and nitrogen fixation in Phaseolus vulgaris. J Gen Microbiol 131, 1779-1786.

Martínez, E., Flores, M., Brom, S., Romero, D., Davila, G. \& Palacios, R. (1988). Rhizobium phaseoli, a molecular genetic view. Plant Soil 108, 179-184.

Martínez-Romero, E., Segovia, L., Mercante, F. M., Franco, A. A, Graham, P. \& Pardo, M. A. (1991). Rhizobium tropici, a novel species nodulating Phaseolus vulgaris L. beans and Leucaena sp. trees. Int J Syst Bacteriol 41, 417-426.

Navarro, E., Simonet, P., Normand, P. \& Bardin, R. (1992). Characterisation of natural populations of Nitrobacter spp. using PCR/RFLP analysis of the ribosomal intergenic spacer. Arch Microbiol 157, 107-115.

Nei, M. \& Li, W. H. (1979). Mathematical model for studying genetic variations in terms of restriction endonucleases. Proc Natl Acad Sci USA 76, 5269-5273.

Nour, S. M., Fernandez, M. P., Normand, P. \& Cleyet-Marel, J. C. (1994). Rhizobium ciceri sp. nov., consisting of strains that nodulate chickpeas (Cicer arietinum L.). Int J Syst Bacteriol 44, 511-522.

Pérez-Ramírez, N. O., Rogel, M. A., Wang, E., Castellanos, J. Z. \& Martínez-Romero, E. (1998). Seeds of Phaseolus vulgaris bean carry Rhizobium etli. FEMS Microbiol Ecol 26, 289-296. 
Piñero, D., Martínez, E. \& Selander, R. K. (1988). Genetic diversity and relationships among isolates of Rhizobium leguminosarum biovar phaseoli. Appl Environ Microbiol 54, 2825-2832.

Ponsonnet, C. \& Nesme, X. (1994). Identification of Agrobacterium strains by PCR-RFLP analysis of pTi and chromosomal regions. Arch Microbiol 161, 300-309.

Pot, B., Gillis, M., Hoste, B., Van De Velde, A., Bekaert, F., Kersters, K. \& De Ley, J. (1989). Intra and intergeneric relationships of the genus Oceanospirillum. Int J Syst Bacteriol 39, 23-34.

Pot, B., Vandamme, P. \& Kersters, K. (1994). Analysis of electrophoretic whole organism proteins fingerprints. In Modern Microbial Methods. Chemical Methods in Prokaryotic Systematics, pp. 493-521. Edited by M. Goodfellow \& A. G. O’Donnell. Chichester: Wiley.

Raven, P. H. \& Polhill, R. M. (1981). Biogeography of the Leguminosae. In Advances in Legume Systematics VI, pp. 27-34. Edited by R. M. Polhill \& P. H. Raven. Kew: Royal Botanic Gardens.

Roberts, G. P., Leps, W. T., Selver, L. E. \& Brill, W. J. (1980). Use of two-dimensional polyacrylamide gel electrophoresis to identify and classify Rhizobium strains. Appl Environ Microbiol 39, 414-422.

Segovia, L., Piñero, D., Palacios, R. \& Martínez-Romero, E. (1991). Genetic structure of a soil population of nonsymbiotic Rhizobium leguminosarum. Appl Environ Microbiol 57, 426-433.

Segovia, L., Young, J. P. W. \& Martínez-Romero, E. (1993). Reclassification of American Rhizobium leguminosarum biovar phaseoli type I strain as Rhizobium etli sp. nov. Int $J$ Syst Bacteriol 43, 374-377.

Selander, R. K., Caugant, D. A., Ochman, H., Musser, J. M., Gilmour, M. N. \& Whittam, T. S. (1986). Methods of multilocus enzyme electrophoresis for bacterial population genetics and systematics. Appl Environ Microbiol 51, 873-884.

Sessitsch, A., Hardarson, G., Akkermans, A. D. \& de Vos, W. M. (1997). Characterization of Rhizobium etli and other Rhizobium spp. that nodulate Phaseolus vulgaris L. in an Austrian soil. Mol Ecol 6, 601-608

Sneath, P. H. A. \& Sokal, R. R. (1973). Numerical Taxonomy. The Principles and Practice of Numerical Classification. San Francisco: Freeman.

Souza, V., Eguiarte Lavila, G., Cappello, R., Gallardo, C., Montoya, J. \& Piñero, D. (1994). Genetic structure of Rhizobium etli biovar phaseoli associated with wild and cultivated bean plants (Phaseolus vulgaris and Phaseolus coccineus) in Morelos, Mexico. Appl Environ Microbiol 60, 1260-1268.

Tjahjoleksono, A. (1993). Caractérisation et diversité des souches de Rhizobium nodulant le haricot (Phaseolus vulgaris) cultivé en trois sites tropicaux. $\mathrm{PhD}$ thesis, Université de Lyon.

Vauterin, L. \& Vauterin, P. (1992). Computer-aided objective comparison of electrophoresis patterns for grouping and identification of microorganisms. Eur Microbiol 1, 37-41.

Vincent, J. M. (1970). A Manual for Practical Study of Root Nodule Bacteria. IBP Handbook No. 15. Oxford: Blackwell.

Weisburg, W. G., Barns, S. M., Pelletier, D. A. \& Lane, D. J. (1991). $16 \mathrm{~S}$ ribosomal DNA amplification for phylogenetic study. $J$ Bacteriol 173, 697-703. 